\title{
The Inter-Relationship of Platelets with Interleukin-1 $\beta$-Mediated Inflammation in Humans
}

\author{
Rahajeng N. Tunjungputri ${ }^{1,2}$ Yang $\mathrm{Li}^{3}$ Philip G. de Groot ${ }^{1}$ Charles A. Dinarello ${ }^{1,4}$ \\ Sanne P. Smeekens ${ }^{1}$ Martin Jaeger ${ }^{1}$ Marije Doppenberg-Oosting ${ }^{1}$ Milou Cruijsen ${ }^{1}$ Heidi Lemmers ${ }^{1}$ \\ Helga Toenhake-Dijkstra ${ }^{1}$ Raul Aguirre-Gamboa ${ }^{3}$ Vinod Kumar ${ }^{3}$ Cisca Wijmenga ${ }^{3,5}$ \\ Leo A. B. Joosten ${ }^{1,6}$ Mihai G. Netea ${ }^{1,7}$ Andre van der Ven ${ }^{1}$ Quirijn de Mast ${ }^{1}$
}

${ }^{1}$ Department of Internal Medicine, Radboud University Medical Center, Nijmegen, The Netherlands

${ }^{2}$ Center for Tropical and Infectious Diseases (CENTRID), Dr. Kariadi Hospital, Faculty of Medicine, Diponegoro University, Semarang, Indonesia

${ }^{3}$ Department of Genetics, University Medical Center Groningen, University of Groningen, Groningen, The Netherlands

${ }^{4}$ Department of Medicine, University of Colorado Denver-Aurora, Colorado, United States

${ }^{5}$ Department of Immunology, K. G. Jebsen Coeliac Disease Research Centre, University of Oslo, Oslo, Norway

6 Department of Medical Genetics, Iuliu Hatieganu University of

Medicine and Pharmacy, Cluj-Napoca, Romania

7 Department for Genomics and Immunoregulation, Life and Medical

Sciences Institute (LIMES), University of Bonn, Bonn, Germany
Address for correspondence Quirijn de Mast, MD, PhD, Department of Internal Medicine, Radboud University Medical Center, Nijmegen, The Netherlands (e-mail: Quirijn.deMast@radboudumc.nl).

\section{Abstract \\ Keywords \\ - platelet immunology \\ - inflammation \\ - infectious diseases \\ - cytokines \\ - platelet physiology}

Background Inflammation and coagulation are key processes in cardiovascular diseases (CVDs). The Canakinumab Anti-inflammatory Thrombosis Outcome Study trial affirmed the importance of inflammation in CVD by showing that inhibition of the interleukin (IL)-1 $\beta$ pathway prevents recurrent CVD. A bi-directional relationship exists between inflammation and coagulation, but the precise interaction of platelets and IL$1 \beta$-mediated inflammation is incompletely understood. We aimed to determine the inter-relationship between platelets and inflammation-and especially IL-1 $\beta$-in a cohort of healthy volunteers.

Methods We used data from the 500-Human Functional Genomics cohort, which consists of approximately 500 Caucasian, healthy individuals. We determined associations of plasma levels of IL-1 $\beta$ and other inflammatory proteins with platelet number and reactivity, the association of platelet reactivity with ex vivo cytokine production as well as the impact of genetic variations through a genome-wide association study (GWAS).

Results Platelets were associated with IL-1 $\beta$ on different levels. First, platelet number was positively associated with plasma IL-1 $\beta$ concentrations $\left(p=8.9 \times 10^{-9}\right)$ and inversely with concentrations of $\alpha$-1-anti-trypsin $\left(p=1.04 \times 10^{-18}\right)$, which is a known antagonist of IL-1 $\beta$. Second, platelet degranulation capacity, as determined by agonistinduced P-selectin expression, was associated with ex vivo IL-1 $\beta$ and IL-6 production. Third, several platelet single-nucleotide polymorphisms (SNPs) were associated with received

June 7, 2018

accepted after revision

September 22, 2018 (c) 2018 Georg Thieme Verlag KG Stuttgart · New York
DOI https://doi.org/

10.1055/s-0038-1675603. ISSN 0340-6245. 
cytokine production and there was a significant platelet SNP enrichment in specific biological important pathways. Finally, platelet SNPs were enriched among SNPs earlier identified in GWAS studies in blood-related diseases and immune-mediated diseases. Conclusion This comprehensive assessment of factors associated with platelet number and reactivity reinforces the important inter-relationship of platelets and IL$1 \beta$-mediated inflammation.

\section{Introduction}

A wealth of pre-clinical and clinical data implicates inflammation as an important driver of ischaemic cardiovascular diseases (CVDs). Until recently, clinical data demonstrating a beneficial effect of inhibiting inflammation were lacking. This has definitely changed with the publication of the results of the Canakinumab Anti-inflammatory Thrombosis Outcome Study (CANTOS) trial, showing that the therapeutic monoclonal interleukin-1 $\beta$ (IL-1 $\beta$ ) antibody canakinumab significantly reduced the risk for recurrent cardiovascular events in highrisk patients who have sustained a prior myocardial infarction. ${ }^{1}$ The pro-inflammatory cytokine IL- $1 \beta$ is a master regulator of inflammation and fever, ${ }^{2}$ and the CANTOS trial affirmed the importance of the IL-1 pathway in atherogenesis. $^{3}$ Nonetheless, the precise processes via which IL-1ß neutralization improves cardiovascular outcome remain unclear. Possible mechanisms include local effects in the atherosclerotic plaque but also systemic actions of IL- $1 \beta$ on platelets and leukocyte mobilization from the bone marrow.

Platelets are increasingly recognized as important effector cells of inflammation and atherosclerosis. ${ }^{4,5}$ Platelets have a multitude of surface receptors that mediate not only haemostatic, but also inflammatory responses. Platelet activation induces the release of granules that contain a diverse array of chemokines, cytokines and growth factors. ${ }^{6}$ Despite being anucleated cells, platelets were suggested to harbour a complex ribonucleic acid (RNA) transcriptome and functional splicing and translational machinery for the synthesis of proteins, ${ }^{7}$ including IL- $1 \beta{ }^{8,9}$

There is considerable variation between individuals in platelet count and platelet reactivity to stimuli. Interestingly, recent data from population-based studies suggest that even variation in platelet count in the range of normality determines future cardiovascular events and mortality. ${ }^{10}$ Variation in platelet count and reactivity is determined by both genetic and nongenetic factors, including inflammation. ${ }^{11,12}$ For example, administration of recombinant IL- $1 \beta$ to patients with gastrointestinal cancer or mice increases platelet count. ${ }^{13,14}$

Several experimental studies have investigated the role of platelets in inflammation and vice versa, but data in humans are limited. We therefore studied the inter-relationship between platelets and inflammation, and especially IL-1 $\beta$, in the 500 Functional Genomics (500FG) cohort, which consists of approximately 500 healthy individuals of Western-European genetic background that is part of the Human Functional Genomics Project (www.humanfunctionalgenomics.org) ${ }^{15}$.
This cohort has the advantage that confounding effects by underlying illness and/or use of drugs are minimal. We first determined the associations of platelet and leukocyte number and platelet reactivity with plasma levels of IL-1 $\beta$ and other inflammatory proteins. Next, we determined associations between platelet reactivity and ex vivo cytokine production as well as the impact of genetic variations through a genomewide association study (GWAS). ${ }^{15}$

\section{Methods}

\section{Functional Genomics Study}

Cohort description: The 500FG study was approved by the Ethical Committee of Radboud University Medical Center (NL42561.091.12, 2012/550). A total of 534 healthy individuals, aged $>18$ years and of Western-European descent, were sampled between July 2013 and December 2014 at the Radboud University Medical Center, The Netherlands. ${ }^{15-18}$ Exclusion criteria were pregnancy/breastfeeding, chronic or acute disease at the time of sampling and use of any medication in the last month before the study.

Measurements of platelet function: Venous blood was collected in citrated tubes (3.2\% sodium citrate; Becton Dickinson, United States) and handled gently to prevent pre-activation. Platelet reactivity was determined by a whole blood flow cytometry assay within 30 minutes as described earlier. ${ }^{19}$ In short, the platelet membrane expression of the $\alpha$-granule protein P-selectin and the binding of fibrinogen to the activated integrin $\alpha_{\text {IIb }} \beta_{3}$ were measured in unstimulated whole blood samples and after ex vivo stimulation by seven increasing concentrations of adenosine diphosphate (ADP, 7.8-125 $\mu \mathrm{M}$, Sigma-Aldrich, United States) or cross-linked collagen-related peptide (CRP-XL, 9-625 ng/mL, kind gift from Prof. Dr. R. Farndale, Cambridge, United Kingdom). The following antibodies were used: phycoerythrin -labelled anti-CD62P (P-selectin; Bio-Legend, San Diego, California, United States), fluorescein isothiocyanate-labelled antifibrinogen (DAKO Ltd., High Wycombe, United Kingdom) and PC7-labelled anti-CD61 (platelet identification marker; Beckman Coulter, France). Samples were analysed using a FC500 flow cytometer and analysed using the CXP software (Beckman Coulter, France). ADP- and CRP-XL-induced platelet P-selectin (termed as APR and CPR) and platelet-fibrinogen reactivity to either ADP or CRP-XL (termed as AFR and CFR) were determined by calculating the area under the curve from the median fluorescence intensity (MFI) of CD62P or fibrinogen on CD61-positive events generated from the 
unstimulated platelets and seven increasing concentrations of ADP or CRP-XL. Plasma concentrations of the platelet $\alpha$ granule protein, $\beta$-thromboglobulin ( $\beta$-TG), a plasma soluble marker for platelet activation, were measured in one batch using enzyme-linked immunosorbent assay (ELISA) as previously described from platelet-poor plasma. ${ }^{20}$

Stimulation of whole blood and peripheral blood mononuclear cells (PBMCS): Full details of these experiments have been detailed elsewhere. ${ }^{16}$ In short, whole blood or PBMCs were co-incubated with the different stimuli for 48 hours (whole blood), 24 hours or 7 days (both PBMCs) at $37^{\circ} \mathrm{C}$ and $5 \% \mathrm{CO}_{2}$. Supernatants were collected and stored at a temperature of $-20^{\circ} \mathrm{C}$. Cytokine concentrations were measured in the supernatant using ELISA.

Measurements of plasma cytokines, acute phase proteins and adipokines: Ethylenediaminetetraacetic acid (EDTA) plasma samples were thawed at room temperature, mixed with an equal volume of specified diluent, centrifuged at $900 \times \mathrm{g}$ for 12 minutes at $4^{\circ} \mathrm{C}$. The diluted plasma supernatants were measured using the Simple Plex cartridges run on the Ella platform (ProteinSimple, San Jose, California, United States) following the manufacturer's instructions. The first step of sample preparation was to perform ultracentrifugation on each sample to remove all particulates and platelets from the plasma. The circulating mediators high sensitive C-reactive protein, leptin, adiponectin and $\alpha-1$ antitrypsin (AAT) were measured in EDTA plasma using the R\&D Systems DuoSet ELISA kits following the manufacture's standard protocol.

\section{In Vitro Validation Experiments}

PBMCs and isolated washed platelets were obtained and experiments were performed as previously described. ${ }^{21}$ In short, PBMCs $\left(3 \times 10^{6}\right.$ cells $\left./ \mathrm{mL}\right)$ isolated from buffy coat (Sanquin Bloodbank, Nijmegen, The Netherlands) were co-cultured for 24 hours at $37^{\circ} \mathrm{C}$ and $5 \% \mathrm{CO}_{2}$ with either washed platelets in a PBMC: platelet ratio of 1:150 or RPMI in the presence of one of the following stimuli: lipopolysaccharides (LPS) $(6 \mathrm{ng} / \mathrm{mL}$; Escherichia coli serotype 055:B5; Sigma-Aldrich), Candida albicans yeast $\left(1 \times 10^{6}\right.$ organisms/mL; UC820), Staphylococcus aureus $\left(1 \times 10^{6}\right.$ organisms/mL; ATCC29213) or E. coli $\left(1 \times 10^{6}\right.$ organisms/mL; ATCC 35218). A Transwell system was used to physically separate platelets and PBMC in some experiments. Platelet-rich plasma (PRP) was isolated from whole blood and was pre-treated with recombinant IL-6 (100 $\mathrm{ng} / \mathrm{mL}$; eBioscience), recombinant IL-1 $(100 \mathrm{ng} / \mathrm{mL}$; R\&D Systems), recombinant interferon (IFN)- $\gamma(100 \mathrm{ng} / \mathrm{mL}$; Boehringer Ingelheim Limited), recombinant IL-18 (10 ng/mL; R\&D Systems) or AAT ( $10 \mu \mathrm{g} / \mathrm{mL}$; Alpha Therapeutic Corporation) for 120 minutes in $37^{\circ} \mathrm{C}$ and $5 \% \mathrm{CO}_{2}$. Platelet reactivity to different platelet agonists was subsequently determined by flow cytometry as described above.

\section{Genotyping and Imputation}

Genotyping in our cohort was performed using the commercially available single-nucleotide polymorphism (SNP) chip, Illumina HumanOmniExpressExome-8 v1.0., and was imputed to obtain genotypes at approximately 7 million
SNPs. The strands and variant identifiers were aligned to the reference Genome of The Netherlands $(\mathrm{GoNL})^{22}$ dataset using Genotype Harmonizer. ${ }^{23}$ The data were phased using SHAPEIT2 v2.r644 ${ }^{24}$ using the GoNL as a reference panel. Finally, these data were imputed using IMPUTE2 ${ }^{25}$ with the GoNL as the reference panel. ${ }^{26}$

\section{Statistical Analysis}

Associations for each measurements of platelet count and platelet function in connection with the acute reactive proteins, adipokines, circulating cytokines and whole blood/PBMC cytokine responses were analysed using the Spearman's correlation coefficient and the statistical significance was based on the false discovery rate (threshold $\alpha \leq 0.1$ ), unless stated otherwise. Analyses were performed using SPSS version 22 .

\section{Results}

\section{The Impact of Non-Genetic Host Factors on Platelets Parameters}

Characteristics of the cohort have been previously described. ${ }^{16-18}$ The median (interquartile range [IQR]) age was 23 years (21-27 years) and $56 \%$ were female. The median body mass index (BMI) was $22.3(20.7-24.4)$ and $83.1 \%$ had a $\mathrm{BMI} \leq 25$. Forty-eight per cent of women used an oral contraceptive and $13.2 \%$ of subjects regularly used tobacco.

Representative platelet reactivity curves, the distribution of these platelet responses and flow cytometry gating strategy are shown in - Supplementary Figs. $\mathbf{S 1}$ and $\mathbf{S 2}$ (available in the online version). Platelet reactivity is expressed as ADPinduced P-selectin expression (APR, available in $n=489$ volunteers) and fibrinogen-binding (AFR, $n=396$ ) and CRP-XL-stimulated P-selectin expression and fibrinogen binding (CPR and CFR, both $n=302$ ). - Fig. 1 illustrates the correlations between platelet parameters. Whereas no correlation was found between unstimulated P-selectin and fibrinogen binding, the four readouts of platelet reactivity correlated well with each other, with the strongest correlations between those in response to the same platelet agonist. There was a positive correlation between plasma concentrations of $\beta$-TG and platelet count, and this was the reason to normalize $\beta$-TG levels for platelet mass in further analyses.

We did not find significant associations between platelet reactivity measures or $\beta$-TG levels with intrinsic, non-genetic host factors such as age, gender, BMI, oral contraceptive usage and smoking. Women had a significantly higher platelet count than men with median (IQR) values of $280(218-352) \times 10^{9} / \mathrm{L}$ versus $251(201-319) \times 10^{9} / \mathrm{L}(p=0.002)$, an observation previously reported by Biino et al. ${ }^{27}$

\section{Associations of Inflammatory Molecules with Platelet Parameters and Leukocyte Count}

We used a novel, high-sensitive cytokine assay capable of measuring very low circulating cytokine concentrations. ${ }^{28}$ Plasma was ultracentrifuged before measurement to remove platelet remnants and other particulates. - Fig. 2A shows associations between these cytokines and platelet parameters. 


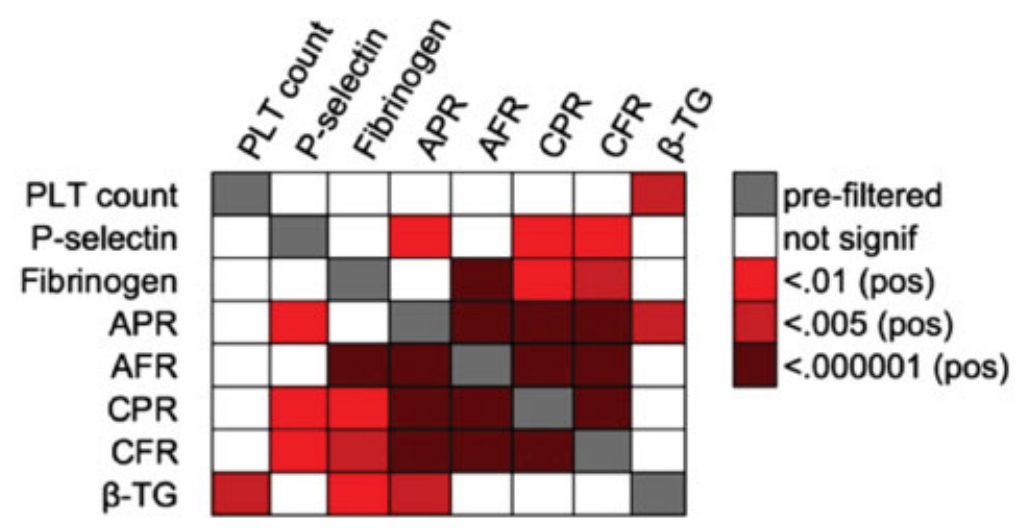

A
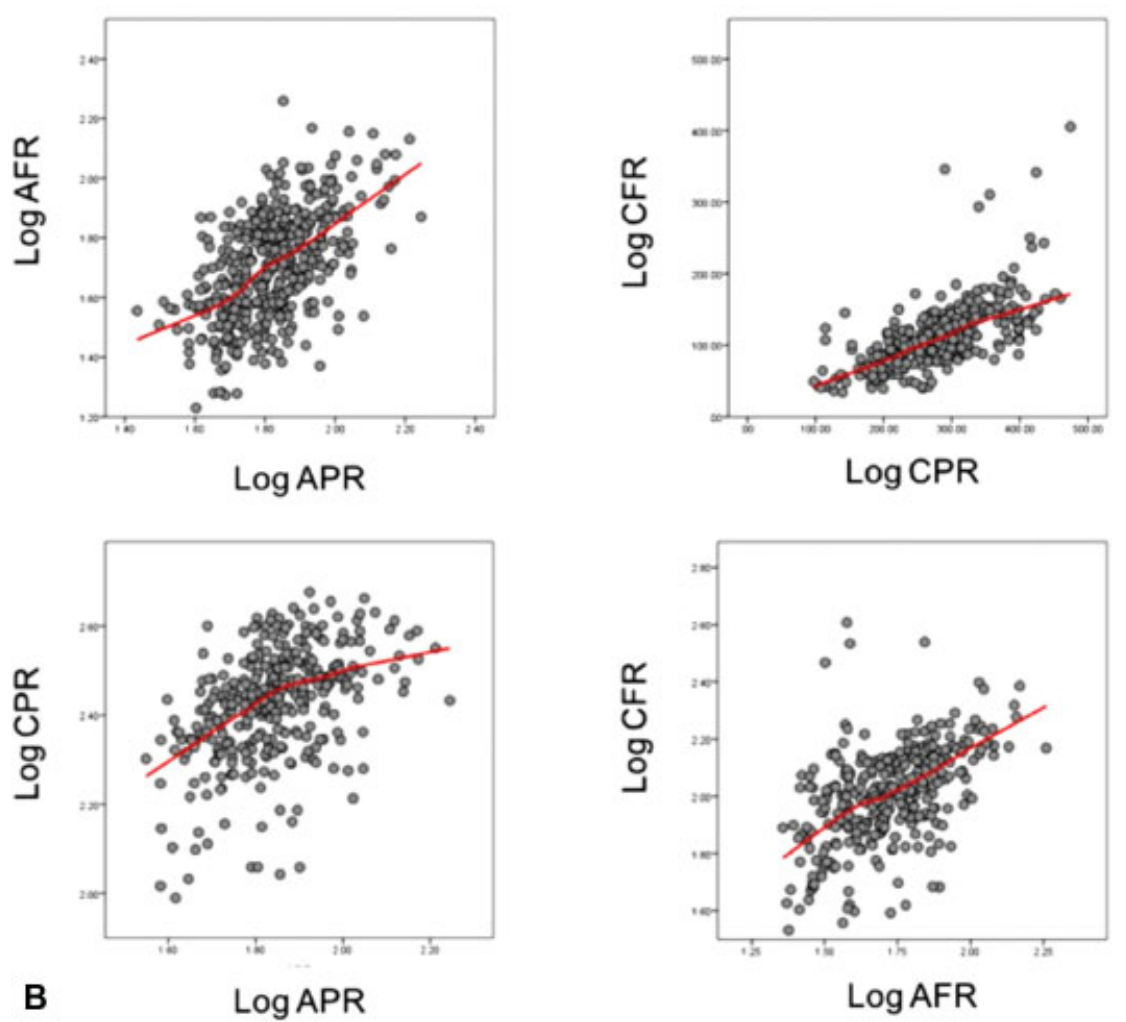

Fig. 1 Correlations between platelet parameters. Data of adenosine diphosphate (ADP)-induced P-selectin expression (APR, available in $n=489$ volunteers) and fibrinogen binding (AFR, $n=396)$ and cross-linked collagen-related peptide (CRP-XL) stimulated P-selectin expression and fibrinogen binding (CPR and CFR, both $n=302$ ) were shown. (A) $p$-Values of the correlations of platelet and coagulation parameters, corrected for multiple testing using the false discovery rate (FDR) method. The colour legend indicates the range of $p$-values. (B) Scatterplots of highly significant correlations from panel (A). The red line shows the LOESS fit to the data. Abbreviations: PLT count, platelet count; P-selectin, unstimulated P-selectin expression; Fibrinogen, unstimulated fibrinogen expression; $\beta$-TG, plasma concentration of $\beta$-thromboglobulin.

The strongest correlation was between plasma IL-1ß concentrations and platelet count (Spearman's $R=0.29$; uncorrected $p=8.9 \times 10^{-9}$ ) and this correlation remained significant after correction for sex, age and use of oral contraceptives using a linear regression model. When volunteers were stratified in tertiles according to their platelet number, those in the highest tertile had a significantly higher median (IQR) IL$1 \beta$ concentrations than those in the lowest tertile $(0.21 \mathrm{pg} / \mathrm{mL}$, 0.1-0.53 $\mathrm{pg} / \mathrm{mL}, \quad$ vs. $\quad 0.09 \mathrm{pg} / \mathrm{mL}, \quad 0.06-0.16 \quad \mathrm{pg} / \mathrm{mL}$; $p<0.0001$ ). Plasma IL-1 $\beta$ were also associated with leukocyte counts $\left(R=0.24 ; p=1.3 \times 10^{-6}\right)$, including neutrophil
$\left(R=0.23 ; p=8.9 \times 10^{-7}\right)$ and monocyte $(R=0.23 ; p=2$ $\times 10^{-6}$ ) count.

Similar to IL-1 $\beta$, platelets were suggested to harbour IL-18 transcripts and synthesize and secrete IL-18 upon activation. They also contain the IL-18 antagonist IL-18 binding protein (IL-18BP). ${ }^{29}$ We found an inverse correlation between platelet number and IL-18BP, as well as a positive association with vascular endothelial growth factor A (VEGF-A), which is also stored in $\alpha$-granules. ${ }^{30}$ In contrast, when plasma cytokine concentrations were related to platelet reactivity, only few weak correlations were present: IL-10 had a negative 


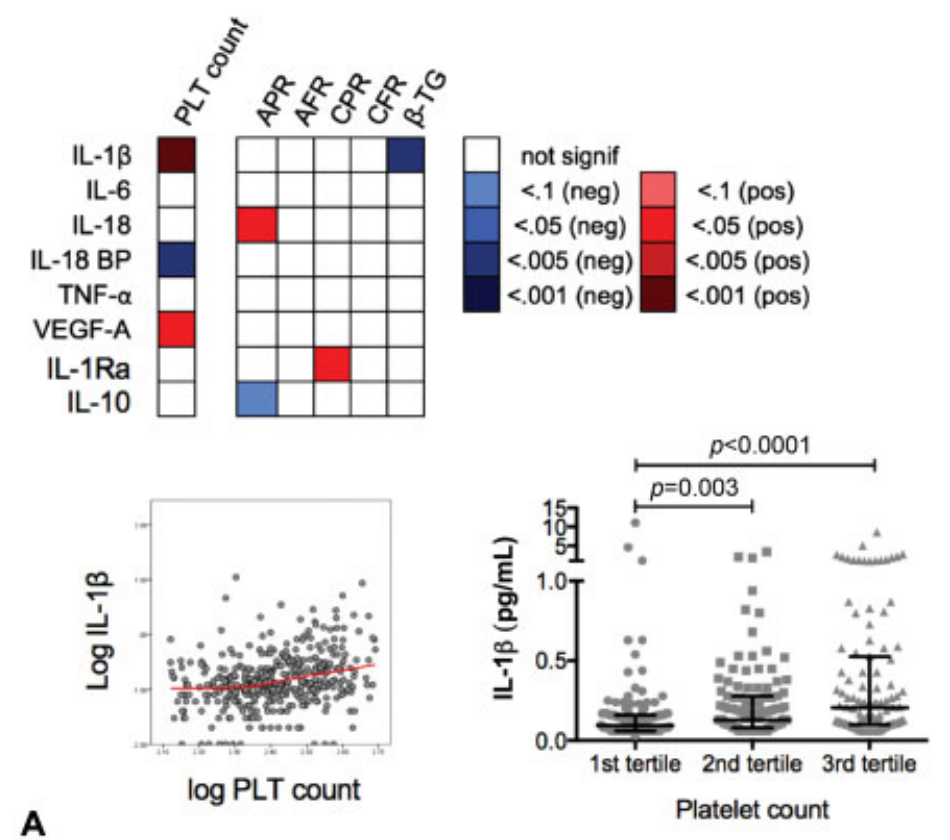

A
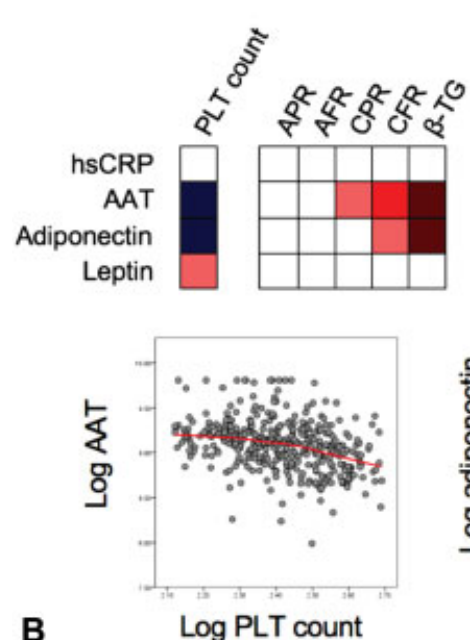

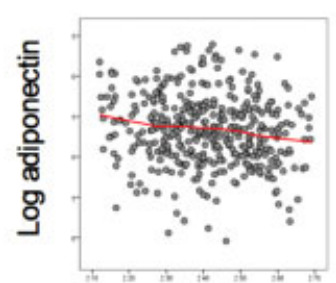

Log PLT count

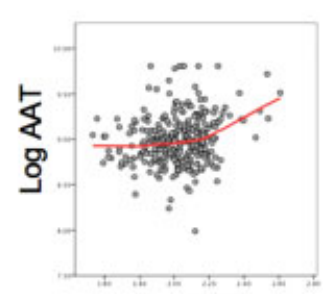

Log CFR

Fig. 2 Correlations of circulating inflammatory mediators with platelet parameters. (A) p-Values of correlations of circulating cytokines with platelet parameters (top panel); scatterplot of the correlation between interleukin (IL)-1 $\beta$ and platelet counts (lower left panel); IL-1 $\beta$ levels in the different platelet count tertiles (lower right panel). (B) $p$-Values (false discovery rate [FDR] corrected) of correlations of acute phase proteins (high sensitive C-reactive protein [hsCRP] and $\alpha 1$-anti-trypsin [AAT]) and adipokines (leptin and adiponectin) with platelet parameters as well as scatterplot of the most significant correlations. The colour legend indicates the range of $p$-values. The red line shows the LOESS fit to the data. Abbreviations: PLT count, platelet count; P-selectin, unstimulated P-selectin expression; Fibrinogen, unstimulated platelet-fibrinogen binding; APR, ADP-induced P-selectin expression; AFR, ADP-induced platelet-fibrinogen binding; CPR, CRP-XL-induced P-selectin expression; CFR, CRP-XLinduced platelet-fibrinogen binding; $\beta$-TG, $\beta$-thromboglobulin $/ 10^{6}$ platelets.

association with APR, IL-18 a positive association with APR and IL-1Ra a positive association with CPR. However, after correction for multiple testing, none of these associations remained statistically significant.

When concentrations of the acute phase proteins C-reactive protein and AAT and the adipokines leptin and adiponectin were related to platelet number and function ( - Fig. 2B), strong negative associations were found between platelet count and AAT $\left(R=-0.398 ; p=1.04 \times 10^{-18}\right)$ as well as adiponectin $\left(R=-0.178 ; p=1.41 \times 10^{-4}\right)$. Both AAT and adiponectin have anti-inflammatory effects and AAT is well known to inhibit IL-1 $\beta,{ }^{31}$ whereas it stimulates IL-1Ra release. ${ }^{32-34}$ Indeed, we found that both AAT and adiponectin correlated inversely with IL-1 $\beta$ concentrations $(R=-$ $0.348 ; p=4.7 \times 10^{-13}$ and $R=-0.146 ; p=0.003$, respectively), but not with IL-1Ra. AAT and adiponectin concentrations were also associated with a higher platelet reactivity to CRP-XL. C-reactive protein concentrations were not associated with any of the platelet parameters. None of the cytokines, acute phase proteins or adipokines correlated with baseline unstimulated P-selectin expression on platelets or platelet-fibrinogen binding.

As platelet counts were higher in women, we analysed the associations between platelet counts and the inflammatory 
molecules separately in males and females. The negative correlations between platelet count and IL-18BP remained in males $(p=0.01)$ and females $(p=0.09)$ despite not being statistically significant for the latter. The negative correlation of platelet count with VEGF-A remained only in females $(p=0.02)$. Plasma levels of cytokines and acute phase proteins in females and males were comparable except for VEGFA and IL-1Ra levels, both higher in women $(p=0.001$ and $p=0.004$, respectively).

\section{Platelet Reactivity is Associated with Cytokine Production Capacity}

We next studied the relationship between platelets and the capacity of immune cells to produce IL-1 $\beta$ and other cytokines. We measured the production of monocyte derived (IL$1 \beta$ and IL-6) and lymphocyte-derived cytokines (IFN- $\gamma$ ) in culture supernatants after ex vivo stimulation of whole blood and PBMCs with the following five stimuli: two purified synthetic microbial ligands: LPS or Pam3CSK4; two bacterial stimuli: E. coli and S. aureus; and one fungal stimulus: C. albicans yeast. Relating platelets to cytokine production by isolated PBMCs is possible because PBMC isolates still contain considerable numbers of platelets. ${ }^{21}$

Platelet P-selectin reactivity to ADP (APR), which corresponds with the release of platelet $\alpha$-granules, was related to IL-1 $\beta$ and IL-6 production to multiple stimuli, whereas CPR was inversely associated with IFN- $\gamma$ production in whole blood ( - Fig. 3A and B). Interestingly, no such associations were found for fibrinogen reactivity, which corresponds more with platelet aggregation, whereas unstimulated fibrinogen binding to $\alpha_{\mathrm{II}} \beta_{3}$ even had few negative associations with cytokine production. Strikingly, while unstimulated P-selectin expression had positive association with IL-6 response to LPS, platelet integrin $\alpha_{\text {II }} \mathrm{b} \beta_{3}$ activity was negatively associated with LPSinduced cytokine responses (-Fig. $\mathbf{3 A}$ ). These observations were validated in a set of in vitro experiments. In line with the observations from our cohort, addition of platelets decreased IFN- $\gamma$ production in response to $C$. albicans and $S$. aureus, whereas IL- $1 \beta$ and IL- 6 responses to all stimuli were significantly increased. Physical separation of PBMC and platelets using a Transwell system reversed these cytokine-modulating effects of platelets ( - Fig. 3C). Conversely, exposure of PRP to recombinant IL-1 $\beta$, IL-6, IFN- $\gamma$ (-Supplementary Fig. S3, available in the online version) and IL-18 (- Supplementary Fig. S4, available in the online version) neither increased Pselectin expression or fibrinogen binding, nor reactivity to ADP or CRP-XL. The exception was AAT, which increased P-selectin expression (- Supplementary Fig. S4, available in the online version).

\section{Genetic Architecture of Platelet Parameters and Relation with Cytokine Production}

We next tested whether we could also demonstrate a relationship between platelets and inflammation at the genetic level. Genetic association studies have identified several SNPs that modify platelet number and reactivity. ${ }^{11,35,36}$ Our genome-wide association analysis did not identify significant hits for the above-mentioned measurements in our cohort. We therefore first performed a pathway analysis using genes in the vicinity of the top platelet quantitative trait locus (QTLs) that had the strongest association with platelet parameters in our cohort (adjusted $p=0.0009$ ). The results indicate that ErbB1, integrin $\beta 1$ receptors, adenosine 5'-diphosphate-ribosylation factor 6 (Arf6), insulin-like growth factor-1 (IGF-1) and platelet-derived growth factor receptors- $\beta$ (PDGFR- $\beta$ ) signalling pathways were identified to be significantly enriched (-Fig. 4). These are important biological pathways, including roles in inflammation, and we have identified in an unbiased way the association with platelets.

We then examined platelet QTLs that were reported in the earlier studies. A list of 133 QTLs was extracted from the literature, including those implicated in platelet number, ${ }^{37-40}$ platelet reactivity ${ }^{11,35,41-44}$ and mean platelet volume (MPV). ${ }^{37,40,45}$ Even though the latter parameter was not measured in our cohort, it was included as platelet size is related to the number of integrins on the platelet membrane ${ }^{11,37}$ and genetic variance in MPV is also associated with platelet reactivity. ${ }^{40}$ Seventy-four QTLs were detected in our cohort and 24 (36\%) of these showed at least one suggestive association with platelet number, platelet reactivity or $\beta$-TG (-Fig. 5). The strongest associations with platelet reactivity were with QTLs in the genes encoding the surface proteins glycoprotein VI (collagen receptor; GP6), ADP receptor P2Y1 (P2RY1), platelet endothelial aggregation receptor 1 (PEAR1), in the gene JMJD1C and in the IL1F10/IL1RN region.

Next, we tested whether the 24 top platelet QTLs (threshold $p=5 \times 10^{-2}$ ) that influence platelet function in our cohort also impacted IL-1 $1 \beta$, IL- 6 and IFN- $\gamma$ cytokine responses in whole blood and PBMC. A sizeable number of these genetic variants were indeed associated with cytokine production: 15 SNPs showed at least marginal association $(p<0.05)$ with one or more cytokine responses, and 5 (rs10761741, rs6734238, rs3794153, rs3091242 and rs11602954) had more than 5 associations (-Fig. 6A). QTLs in the genes of the surface receptors GP6 and P2YR1 and in PEAR1 or JMJD1C, which all had strong associations with platelet reactivity, had either no or only suggestive association with cytokine production. Interestingly, strong, negative associations with cytokine responses was found for rs6734238, a genetic variant located downstream of IL1F10 and upstream of IL1RN, which encodes the anti-inflammatory cytokine IL-1Ra. ${ }^{46}$ This QTL also correlated inversely with AFR in our cohort.

Lastly, we tested for associations in the opposite direction, that is, whether several QTLs that we recently found in the same cohort to be related with cytokine responses ${ }^{47}$ were associated with platelet responses ( $\mathbf{- F i g . 6 B}$ ). Only few associations were found. Again, a QTL in a locus near IL1RN (rs4496335) was inversely related with AFR, whereas rs10908219, which is located near the genes for fibroblast growth factor (FGF)-19, FGF4 and FGF3 were positively associated with ADP- and CRP-XL-induced $\alpha_{\text {IIb }} \beta_{3}$ integrin activation. Finally, given the association of platelet count with plasma IL-1 $\beta$ concentrations, we assessed whether the IL- $1 \beta$ pathway was enriched for platelet genes. However, no shared SNPs were found for platelet count and IL-1ß. 


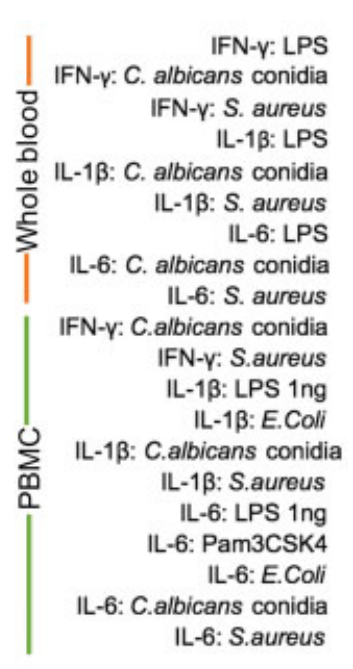

A

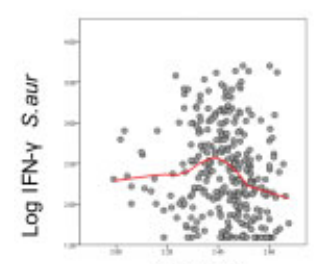

$\log$ CPR

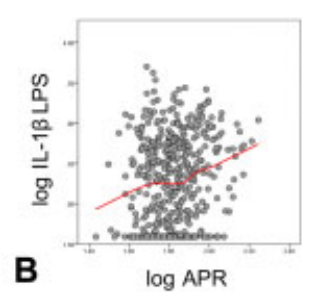

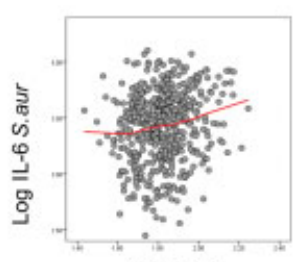

$\log$ APR

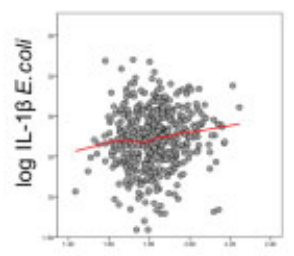

$\log$ APR

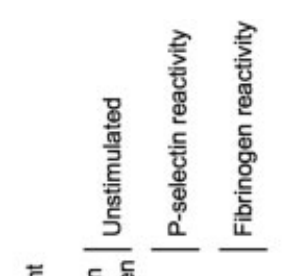

\section{은}
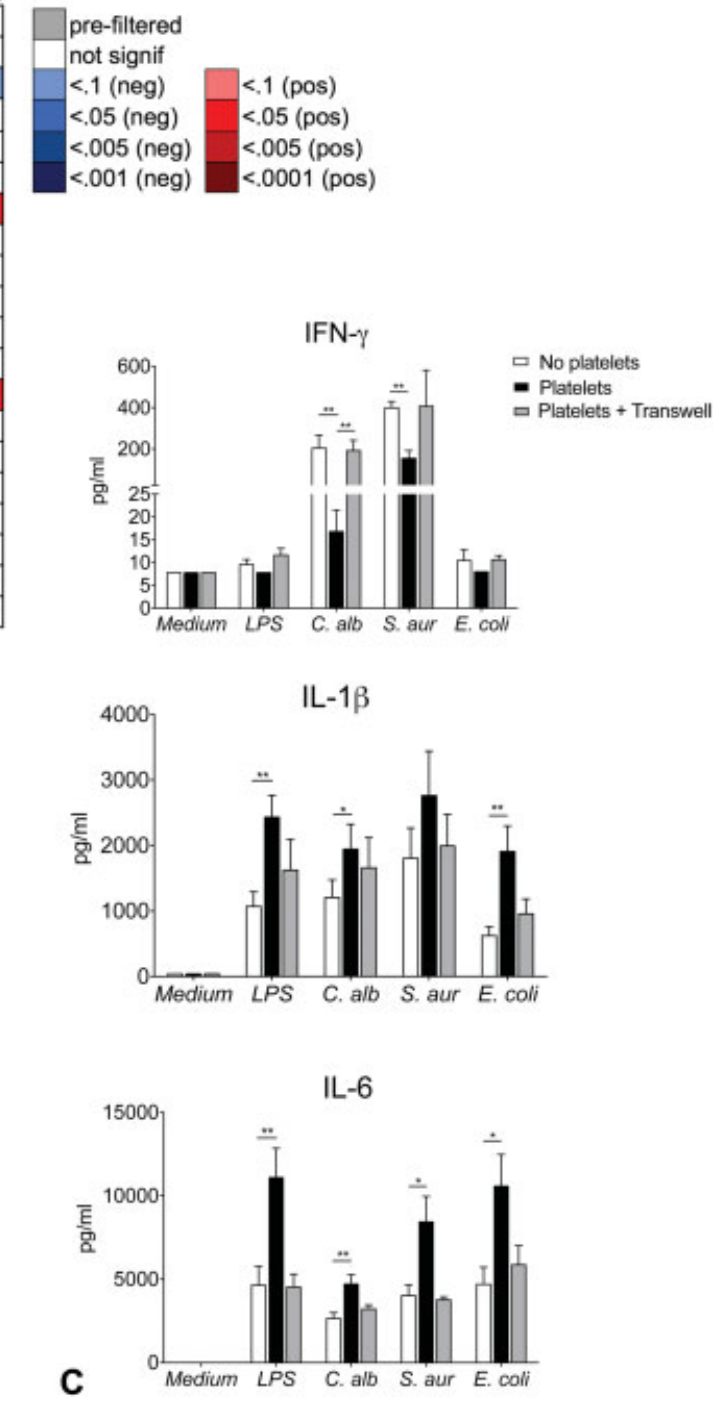

Fig. 3 Associations of platelets with cytokine responses. (A) $p$-Values (false discovery rate [FDR] corrected) of the correlations of whole blood and peripheral blood mononuclear cells (PBMCs) cytokine responses with platelet parameters. The colour legend indicates the range of $p$-values. (B) Scatterplots of the highly significant correlations of whole blood cytokine responses from panel A. The red line shows the LOESS fit to the data. (C) PBMCs ( $3 \times 10^{6} \mathrm{cells} / \mathrm{mL}$ ) were co-cultured with either washed platelets or RPMI and the following stimuli for 24 hours: lipopolysaccharides (LPS) $(6 \mathrm{ng} / \mathrm{mL}), \mathrm{C}$. albicans yeast $\left(1 \times 10^{6}\right.$ organisms $/ \mathrm{mL})$, S. aureus $\left(1 \times 10^{6}\right.$ organisms $\left./ \mathrm{ml}\right)$ and $E$. coli $\left(1 \times 10^{6}\right.$ organisms $\left./ \mathrm{mL}\right)$ with or without physical separation between platelets and PBMC using a Transwell system. Interleukin (IL)-1 $\beta$, IL-6 and interferon (IFN)-y concentrations were determined in the culture supernatant. Platelets were added in a ratio of 150 platelets for every PBMC. Presented data are means with standard error of the mean (SEM) from 6 healthy donors. ${ }^{*} p<0.05,{ }^{* *} p<0.01,{ }^{* * *} p<0.005 . C$. alb, Candida albicans; S. aur, Staphylococcus aureus; PLT count, platelet count; P-selectin, unstimulated P-selectin expression; Fibrinogen, unstimulated plateletfibrinogen binding; APR, ADP-induced P-selectin expression; CPR, CRP-XL-induced P-selectin expression; $\beta$-TG, $\beta$-thromboglobulin $/ 10^{6}$ platelets.

\section{Platelet SNPs Overlap with SNPs Associated with Immune-Mediated Diseases}

Finally, we intersected our platelet QTLs with the information of GWASs for various diseases available in the literature. We extracted GWAS SNPs from the National Human Genome
Research Institute GWAS catalog 48 and binned them into eight categories on the basis of their association with different human phenotypes (cancer, immune-mediated diseases, infectious disease, heart-related traits, blood-related traits, metabolic traits, height and type 2 diabetes-related traits). 

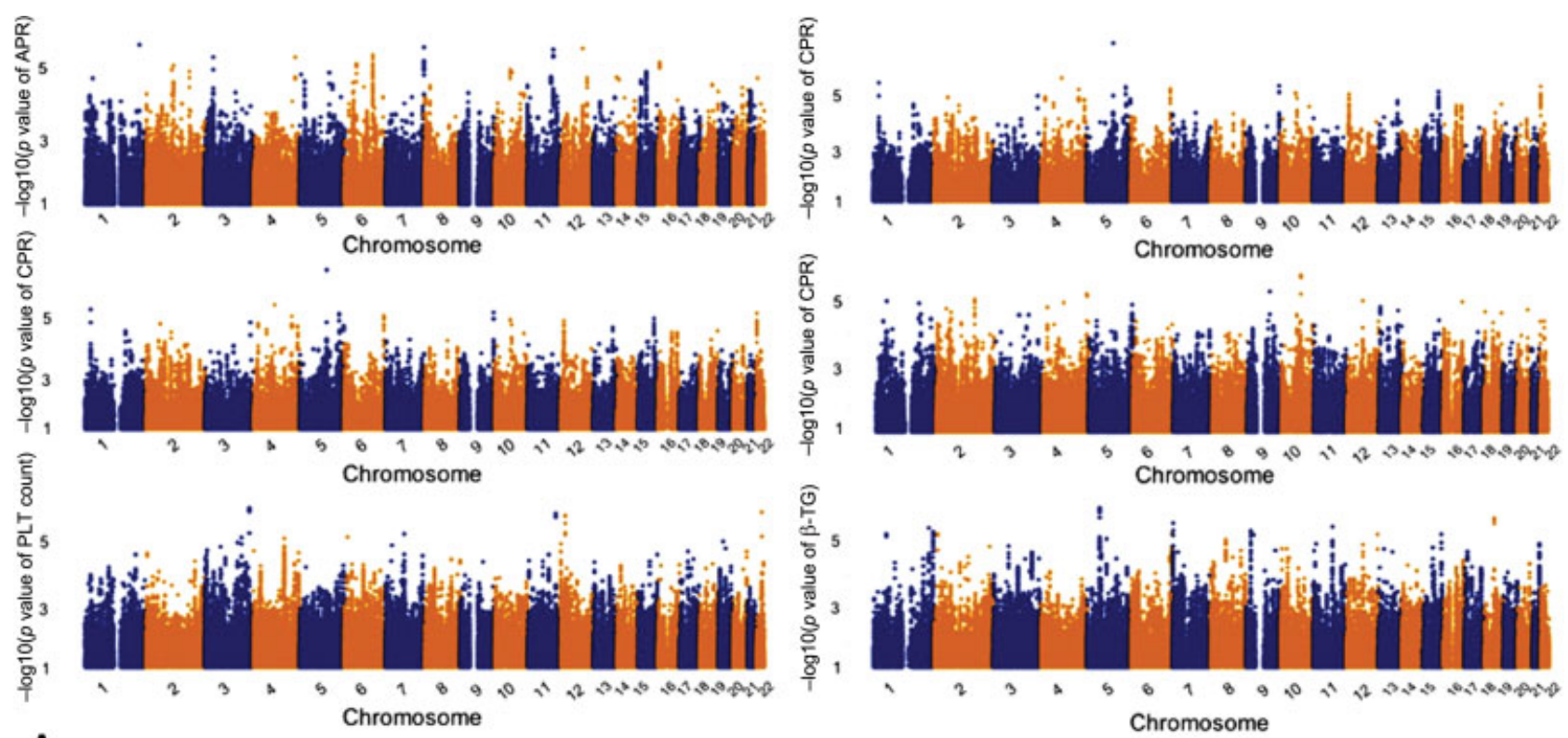

A

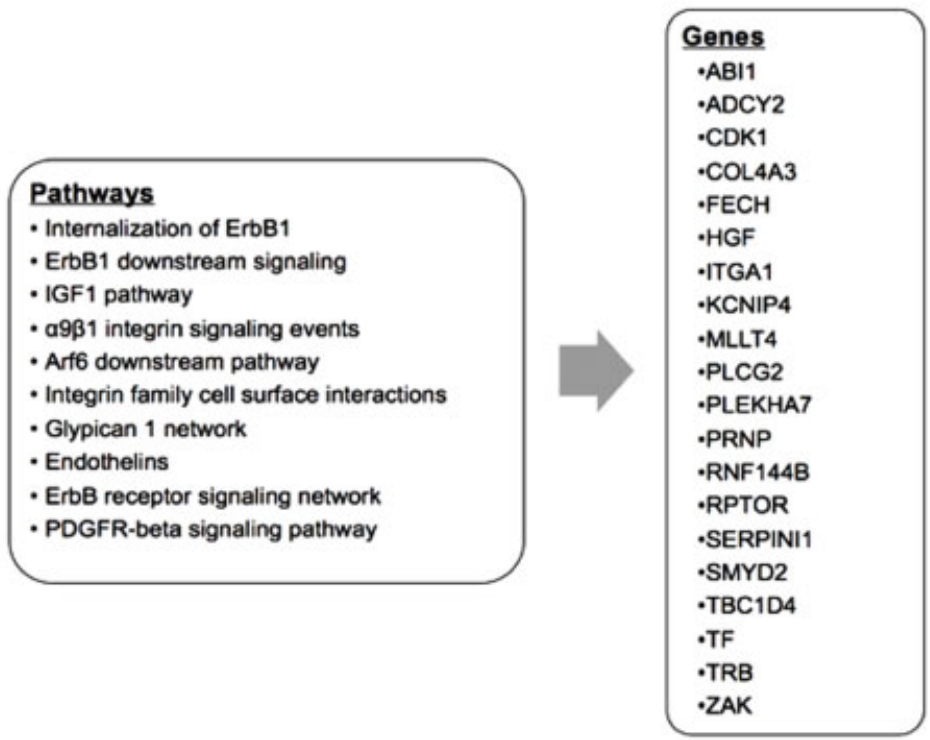

Fig. 4 Genome-wide quantitative trait locus (QTL) mapping for platelet phenotypes. (A) Manhattan plots showing the genome-wide QTL mapping results for APR, CRP, AFR, CFR, platelet count and $\beta$-TG. The vertical axis in the Manhattan plots indicates (-log10 transformed) observed $p$-values. QTLs are considered as reaching genome-wide significance at the level of $p=5 \times 10^{-8}$. (B) Lists of pathways and genes enriched for platelet QTLs.

Next, we identified all QTLs that were associated with platelets at $p<1 \times 10^{-4}$ and tested whether they are also GWAS QTLs or their proxies $\left(r^{2}>0.8\right)$. Platelet QTLs are enriched among SNPs earlier identified in GWASs in bloodand immune-mediated diseases, suggesting the role for platelets in the pathogenesis of those diseases ( - Fig. 7). In contrast, no enrichment was found for heart-related diseases or infections.

\section{Discussion}

In this study, we report a comprehensive assessment on the functional and genomic architecture of platelet number and reactivity, and the relation with inflammation and cytokine production in a healthy human population. We report that IL-
$1 \beta$ plasma levels are associated with platelet number and that platelet reactivity is associated with inflammatory markers and IL-1 $\beta$ and IL-6 production capacity. We also found that several crucial signalling routes (e.g. ErbB1, PDGFR- $\beta$ and IGF-1 pathways) are enriched for platelet SNPs, and that SNPs that influence platelet number and function are enriched in GWASs on haematologic and immune-mediated diseases.

Platelets are long known to play an important role in inflammation, including the onset and progression of atherosclerosis. $^{5}$ The results of the CANTOS trial now offer important new evidence for a key role of IL-1 $\beta$ in atherogenesis and cardiovascular events. Our study shows for the first time in a large cohort of healthy, predominantly young adults that platelets and IL-1 $\beta$ are related on different levels: platelets numbers are associated with IL-1 $\beta$, but not with IL-6 or 
<smiles>C1C2CC1C2</smiles>

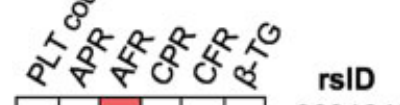

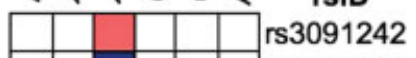

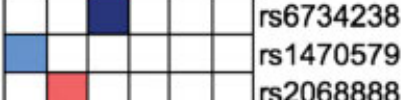
\begin{tabular}{|l|l|l|l|lll}
\hline & & & & & & rs206888 \\
\hline & & & & & & rs3794153 \\
\hline
\end{tabular}

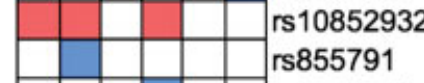

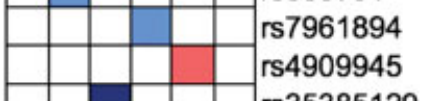

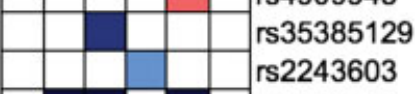
\begin{tabular}{|l|l|l|l|l}
\hline & & & & \\
\hline & $r 232393967$ \\
\hline
\end{tabular}

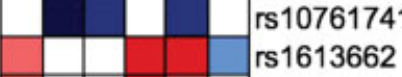
rs 1671152 rs11264579 rs17786144 rs7940646 rs4311994 rs3755711 rs701265 rs3821667 rs2046934 rs 1388626 rs6809699 rs10883735 rs9889955

Locus
TMEM50A
IL1F10/IL1RN
IGF2BP2
EXOC6
ST5
SMG6
TMPRSS6
WDR66
MRVI1
PVR
SIRPB1
JMJD1C
JMJD1C
GP6
GP6
PEAR1
ITPR1
MRVI1
ADRA2A
P2RY1
P2RY1
P2RY12
P2RY12
P2RY12
P2RY12
SUFU
SDK2

Previously-reported association
Platelet count
Platelet count
Platelet count
Platelet count
Platelet count
Platelet count
Platelet count
MPV, Platelet count
MPV
MPV
MPV
MPV

Platelet count, epinephrine-induced light aggregation, MPV CPR, CFR

Collagen-induced aggregation

APR, AFR

APR, AFR

ADP-induced light aggregation

Epinephrine-induced light aggregation

AFR

AFR

AFR

AFR

AFR

AFR

ADP-induced light aggregation

ADP-induced light aggregation

Fig. 5 Associations between known platelet single-nucleotide polymorphisms (SNPs) from the literature and platelet parameters in our cohort. The colour legend indicates the range of $p$-values. $p$-Values were obtained from a linear regression model of platelet parameters on genotype data. APR, ADP-induced P-selectin expression; AFR, ADP-induced platelet-fibrinogen binding; CPR, collagen-induced P-selectin expression; CFR, collagen-induced platelet-fibrinogen binding. APR, AFR, CPR and CFR were data obtained from whole blood flow cytometry. MPV, mean platelet volume.

tumor necrosis factor- $\alpha$, and platelet degranulation capacity was associated with IL-1 $\beta$ cytokine production capacity of the leukocytes. The latter may especially be important locally in the atherosclerotic plaque. These findings reinforce the notion that platelets are integrated with the function of the immune system, ${ }^{4,49}$ and that not only the thrombotic but also the inflammatory function of platelets is important in atherothrombosis. Similarly, they suggest that part of the results of CANTOS may be due to effects of IL-1 $\beta$ inhibition on platelets production, but not on platelet reactivity. The administration of human recombinant IL- $1 \beta$ was previously demonstrated to increase platelet numbers in patients with gastrointestinal cancer ${ }^{13}$ and in mice. ${ }^{14}$ Interestingly, thrombocytopaenia was significantly more common in patients in the canakinumab groups in the CANTOS trial compared with the placebo group. Our findings in healthy volunteers therefore support that IL-1 $\beta$ is a regulator of platelet number. ${ }^{50}$ Platelets were also reported to have the capacity to synthesize and secrete IL- $1 \beta$, the latter primarily within micro-particles. ${ }^{8,9,51}$ Due to their high circulating number (150-400.000/ $\mu \mathrm{L}$ blood), platelets may as such be a relevant source for IL-1 $\beta$. This creates a feedback loop whereby IL-1 $\beta$ induces thrombopoiesis which in turn leads to more platelet- derived IL-1 $\beta$. The strong inverse correlation of AAT with platelet number can be explained by the fact that AAT inhibits the expression of IL- $1 \beta$ and other pro-inflammatory cytokines. $^{31}$

Platelets themselves do not contain mature IL- $1 \beta$ but are capable of its release through signal-dependent splicing of intra-platelet IL-1 $\beta$ pre-messenger RNA (mRNA). Although measurements of platelet-derived IL- $1 \beta$ were not performed in our 500FG study, evidences were available from several other studies. IL- $1 \beta$ synthesis and inflammasome assembly in platelets are initiated in response to pro-coagulant or pathogen-associated stimuli. ${ }^{52,53}$ Signal-dependent splicing of IL-1 $\beta$ pre-mRNA in platelets is regulated by cdc-like Kinase 1 (CLK1 $)^{51,54}$ and depends on activation by thrombin or other classic agonists in the presence of fibrinogen and requires outside-in signalling from integrin activation. ${ }^{9,51}$ CLK1mediated IL-1 $\beta$ RNA splicing also occurs after Toll-like receptor (TLR)-2- and TLR-4-mediated platelet activation by Pam3Cys or LPS, respectively. ${ }^{51,54}$ Inflammasomes are also one source of IL- $1 \beta$. Furman et al showed that the nucleotide-derived metabolites adenine and N4-acetylcytidine, which were detectable in the blood of older individuals with constitutive expression of IL-1 $\beta$, primed and activated 

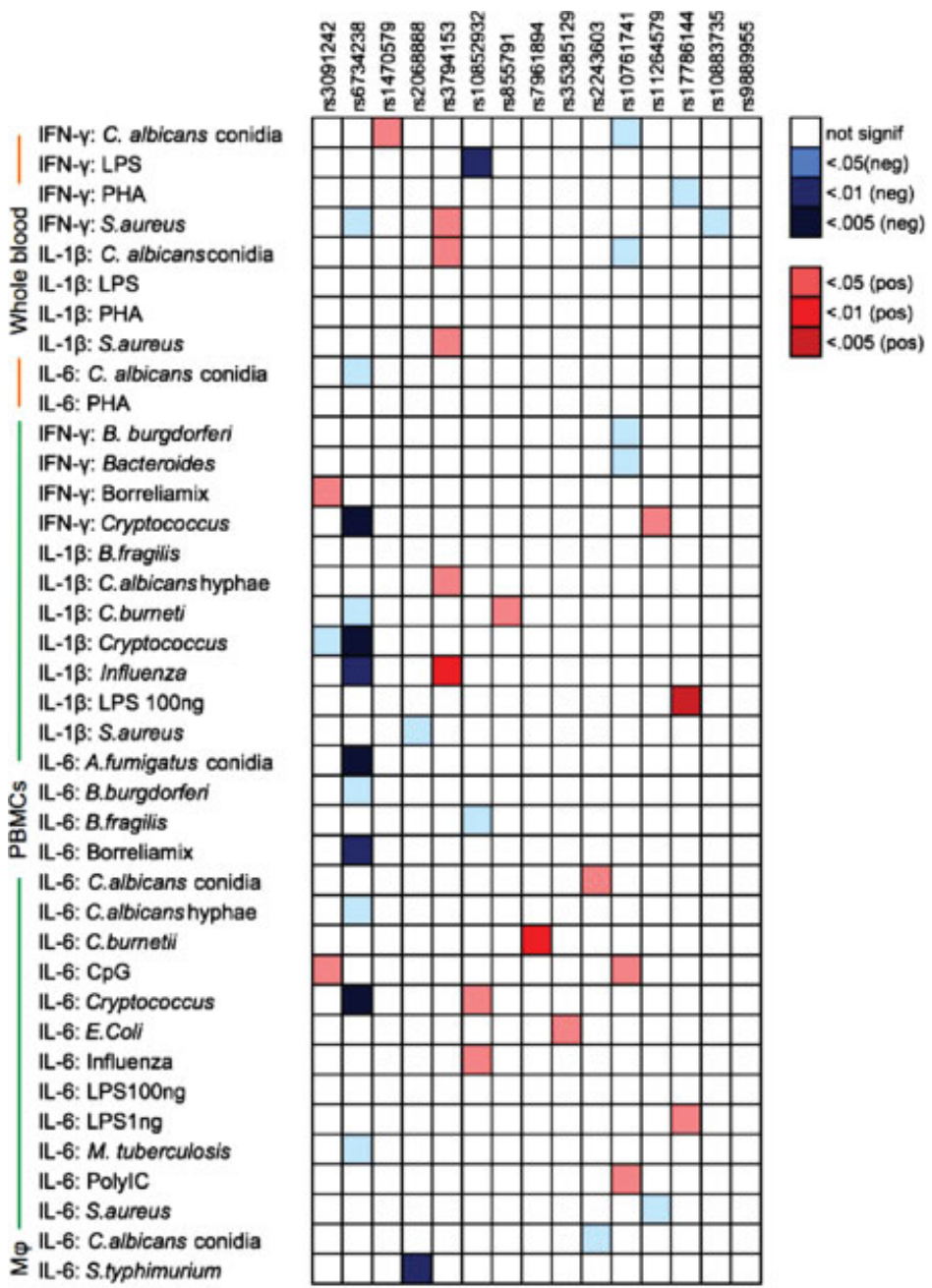

A

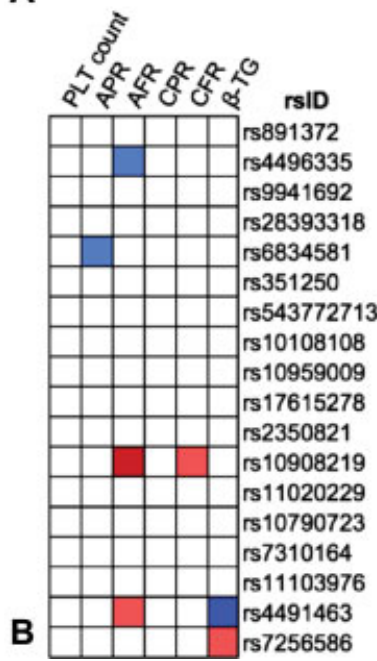

\begin{tabular}{|c|c|c|}
\hline \multirow{2}{*}{$\begin{array}{l}\text { Locus } \\
\text { CD55, CR2 }\end{array}$} & \multicolumn{2}{|c|}{ Previously-reported association } \\
\hline & C.burnetii & IL-6 \\
\hline IL $1 R N$ & Cryptococcus & TNF- $\alpha$ \\
\hline FMNL2, STAM2 & C.bumetii & IL-6 \\
\hline TLR1, TLR6, TLR10, FAM114A1 & Poly(l:C) & $\mathrm{IL}-1 \beta$ \\
\hline TLR1, TLR6, TLR10, FAM114A1 & C.bumetii & $\mathrm{IL}-1 \beta$ \\
\hline SLC25A2, PCDH12, KIAA0141 & C.bumetii & IL-6 \\
\hline ZNF12 & C. albicans & TNF- $\alpha$ \\
\hline CSMD1 & C. albicans & $\mathrm{IL}-22$ \\
\hline PTPRD & C. burnetii & IL-6 \\
\hline RP11-92J19.3, RP11-810B23.1 & Borrelia & IFN-y \\
\hline$R P 11-181 F 12,1$ & LPS & $\mathrm{IL}-1 \beta$ \\
\hline FGF19, FGF4, FGF3, MIR3164 & Cryptococcus & IFN-Y \\
\hline SLC36A4 & S. aureus & $\mathrm{IL}-22$ \\
\hline SLC37A2, AP001007.1 & C.bumetii & $\mathrm{IL}-1 \beta$ \\
\hline ITGA7, CD63,MMP19 & C.bumetii & IL-6 \\
\hline MGAT4C & Borrelia & IFN-y \\
\hline$R P 11-47513.1, R P 1147513.2$ & E. coli & TNF- $a$ \\
\hline$K L K 2, K L K 4$ & C. albicans & IL-6 \\
\hline
\end{tabular}

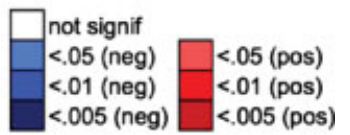

Fig. 6 Single-nucleotide polymorphisms (SNPs) associated with overlapping platelet traits and cytokine production capacity in whole blood and peripheral blood mononuclear cells (PBMCs). (A) p-Values of the associations of cytokine responses with platelet SNPs; $p$-Values determined from linear regression analysis of cytokine responses on genotype data. (B) $p$-Values of the associations of platelet parameters with cytokine SNPs. $p$ Values were from linear regression analysis of platelet parameters on genotype data. The colour legend indicates the range of $p$-Values.

the NOD-like receptor family CARD domain-containing protein 4 inflammasome, induced the production of IL-1ß, activated platelets and neutrophils and elevated blood pressure in mice. ${ }^{55}$ Additionally, dengue virus infection was reported to lead to the assembly of nucleotide-binding domain leucine-rich repeat containing protein (NLRP3) inflammasomes, activation of caspase- 1 and caspase-1dependent IL-1 $\beta$ secretion. This study also indicated that 


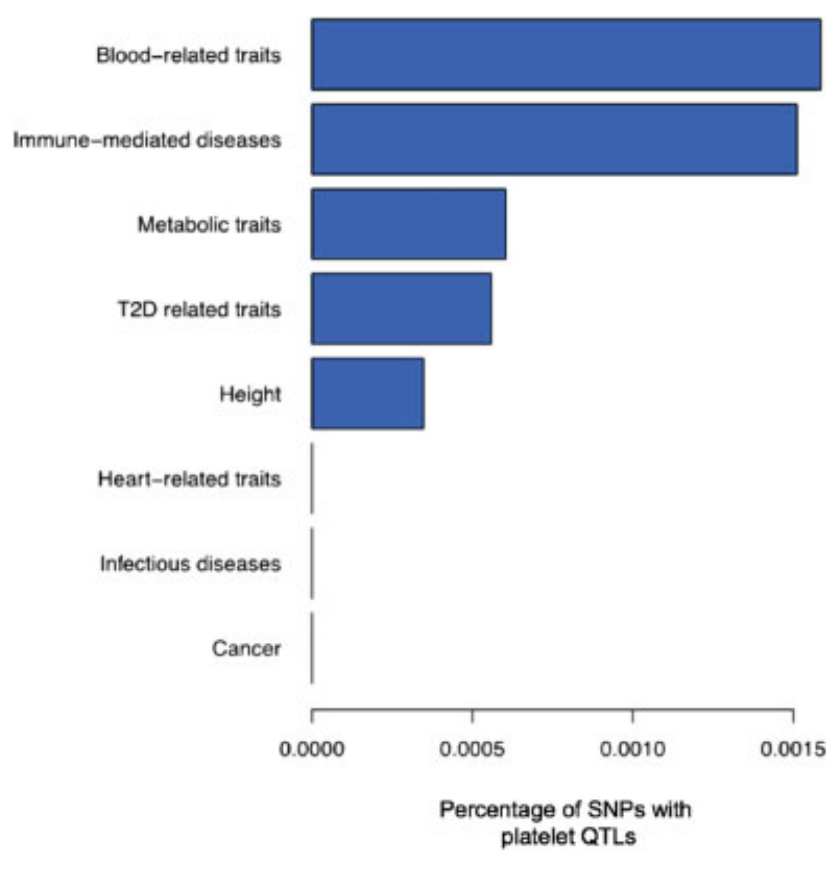

Fig. 7 Platelet quantitative trait locus (QTLs) overlap with singlenucleotide polymorphisms (SNPs) associated with immune-mediated diseases. The percentage of SNPs associated with each category of disease that also qualified as suggestive platelet QTLs.

platelet-derived IL-1 $\beta$ is released in micro-particles through mechanisms dependent on mitochondrial reactive oxygen species-triggered NLRP3 inflammasomes. ${ }^{8}$

The effects of IL-1 $\beta$ on the bone marrow were not restricted to platelets, as plasma IL- $1 \beta$ levels were also strongly associated with leukocyte counts, including neutrophils and monocytes. Similar to thrombocytopaenia, leukopenia was also more common in the canakinumab-treated groups in the CANTOS trial. Leukocytes are also key cells in the aetiology of atherosclerosis and cardiovascular events, and inhibition of IL-1 $\beta$-mediated leukocyte mobilization from the bone marrow may further explain the results of canakinumab.

Whereas platelet degranulation capacity, as measured by P-selectin reactivity, was positively associated with ex vivo IL-1 $\beta$ and IL-6 production, these cytokines had little effect on platelet reactivity. This largely one-directional relationship whereby more reactive platelets boost IL-1 $\beta$ and IL- 6 production is supported by earlier studies that, even though platelets contain a functional IL- 1 receptor, IL-1 $\beta$ and LPS act as atypical platelet activators. ${ }^{53}$ In contrast, others have found that IL-1 $\beta$ increases platelet aggregation. ${ }^{50}$ IFN- $\gamma$ inhibits IL- $1 \beta$ production, ${ }^{56}$ which may be a possible explanation for the opposite associations between platelet reactivity with primarily monocyte-derived (IL-1 $\beta$ and IL-6) and T-lymphocyte-derived (IFN- $\gamma$ ) cytokines. Platelet micro-particles may also impair differentiation of regulatory T-cells into IL-17 and IFN- $\gamma$-producing cells, and as such inhibit IFN$\gamma$ production. ${ }^{57}$ In contrast to the positive associations of platelet degranulation (P-selectin) capacity with cytokine production, we speculate that platelet integrin $\alpha_{\mathrm{IIb}} \beta_{3}$ activity inhibits cytokine responses. The fact that platelets can attenuate cytokine responses to LPS has been reported recently. 58

Platelet degranulation may be inter-linked with cytokine production in different ways: (1) the ligation of platelet Pselectin with monocyte P-selectin glycoprotein ligand-1 triggers nuclear translocation of nuclear factor-kappa $B$ (NF-KB) and activation of the NF-KB-dependent inflammatory genes in monocytes, ${ }^{59}$ as observed in our current and prior experiments using TLR4 ligand ${ }^{21}$ (2) platelet phagocytosis by PBMCs may lead to concomitant surface TLR internalization or platelet microRNA-dependent cytokine downregulation in leukocytes ${ }^{21,58}$ (3) platelets contain a large array of granule-stored chemokines and cytokines, and when stimulated, platelets also produce inflammatory molecules de novo ${ }^{9,51}$; and (4) genetic overlap exists in pathways involved in platelet activation and cytokine production, for example, JMJD1C. Interestingly, whereas genetic variation in surface platelet receptors (such as P2Y12 and GPVI) had no effect on cytokine production, two QTLs in a locus near IL1RN (rs6734238 and rs4496335) were inversely related to ADPinduced fibrinogen reactivity.

The GWAS also enabled us to show enrichment of platelet QTLs in different biologically important pathways, including the ErbB1, PDGFR- $\beta$, Arf6, ${ }^{60}$ IGF1 ${ }^{61}$ and integrin $\beta 1$ receptors pathways. ErbB1 and PDGFR are important regulators of cell proliferation, survival, differentiation and migration. ${ }^{62,63}$ The other pathways had been more directly associated with platelets: Arf6 protein was recently reported to control platelet spreading and clot retraction via integrin $\alpha_{\mathrm{IIb}} \beta_{3}$ trafficking ${ }^{60}$; the integrin $\beta 1$ signalling is required for platelet granule secretion and hemostasis ${ }^{64}$; and IGF-1, released from the $\alpha$-granules upon platelet activation, potentiates platelet aggregation. ${ }^{61}$ Some of these pathways are also important in inflammation and the importance of platelets in these pathways is thus independently confirmed by our study using this unbiased approach. In addition, there was a strong enrichment of platelet QTLs in GWASs identifying SNPs that impact haematologic and immune-mediated disorders. The latter is not surprising given the role of platelets in inflammation and immune-mediated illnesses. For example, platelets have been implicated in the pathogenesis of rheumatoid arthritis. ${ }^{65,66}$ There was much less enrichment of platelet QTLs in the SNP dataset identified in GWASs to increase susceptibility and control to a selection of infections. These infections, however, included hepatitis B, hepatitis $C$, human immunodeficiency virus, smallpox, leprosy and malaria, and platelets are less likely to play a role in the pathophysiology of these specific infections.

Finally, of the non-genetic factors, the limited effect of gender and age on platelet function suggests that variability of platelet function based solely on age or sex is not a major factor in the known difference of platelet-dependent pathologies in these groups. Interestingly, smoking or BMI also did not have a major impact on platelets in these predominantly young, healthy individuals. Although smokers were previously reported to have increased platelet aggregation response, this was only found transiently after smoking ${ }^{67}$ and/or in those with underlying coronary artery disease. ${ }^{68}$ 
Previous studies also did not find any association between obesity and platelet activation. ${ }^{69}$

Our study has different limitations. First, measurements of CRP-XL-induced platelet reactivity were only available in 302 individuals. Second, the associations presented here do not necessarily imply causality, although many of the significant associations, such as platelet number and IL- $1 \beta$, are supported by data from earlier pre-clinical studies. Finally, whereas the enrolment of young, healthy volunteers without co-morbidities is a particular strength of our studies, it may not reflect the common and important relations between platelets and inflammatory parameters as may be observed in a diseased setting.

In conclusion, we comprehensively describe the pattern of interactions of the number and function of platelets with host factors, genetic variation and inflammation and immune responses. We demonstrate the association between platelets and IL-1 $\beta$-mediated inflammation as well as circulating leukocyte numbers, thereby adding important novel insights in how IL-1 $\beta$ neutralization may protect against cardiovascular events.

\section{What is known about this topic?}

- Platelets are key cells in inflammation and host defence.

- Interleukin (IL)-1 $\beta$ inhibition was recently shown to prevent against recurrent cardiovascular events.

\section{What does this paper add?}

- IL-1 $\beta$ plasma levels are strongly associated with platelet number in healthy human individuals, but not with platelet reactivity.

- Platelet reactivity is associated with IL-1 $\beta$ and IL-6 production capacity.

- Several crucial signalling routes in inflammation are enriched for platelet SNPs.

\section{Funding}

The Human Functional Genomics Project is supported by a European Research Council (ERC) Consolidator grant (ERC 310372). R.N.T. is an awardee of the DIKTI-NESO PhD fellowship from the Ministry of Education of Indonesia. C. A.D. is supported by NIH Grant AI-15614. This study was further supported by an IN-CONTROL CVON grant (CVON2012-03) and a Netherlands Organization for Scientific Research (NWO) Spinoza prize (NWO SPI 94-212) to M. G.N; by a an ERC Advanced grant [FP/2007-2013/ERC grant 2012-322698] and an NWO Spinoza prize [NWO SPI 92266] to C.W.; a European Union Seventh Framework Program grant (EU FP7) TANDEM project [HEALTH-F3-2012305279] to C.W. and V.K.; and a Research Grant [2017] of the European Society of Clinical Microbiology and Infectious Diseases (ESCMID) to V.K.
Conflict of Interest

None.

\section{Acknowledgements}

The authors thank all of the volunteers in the 500FG cohort for their participation. The authors also thank Isak W. Tengesdal for performing the cytokine measurements using the Ella apparatus (Protein Simple).

\section{References}

1 Ridker PM, Everett BM, Thuren T, et al; CANTOS Trial Group. Antiinflammatory therapy with canakinumab for atherosclerotic disease. N Engl J Med 2017;377(12):1119-1131

2 Dinarello CA. Interleukin-1 in the pathogenesis and treatment of inflammatory diseases. Blood 2011;117(14):3720-3732

3 Libby P. Interleukin- 1 beta as a target for atherosclerosis therapy: biological basis of CANTOS and beyond. J Am Coll Cardiol 2017;70 (18):2278-2289

4 Semple JW, Italiano JE Jr, Freedman J. Platelets and the immune continuum. Nat Rev Immunol 2011;11(04):264-274

5 Davì G, Patrono C. Platelet activation and atherothrombosis. N Engl J Med 2007;357(24):2482-2494

6 Vieira-de-Abreu A, Campbell RA, Weyrich AS, Zimmerman GA. Platelets: versatile effector cells in hemostasis, inflammation, and the immune continuum. Semin Immunopathol 2012;34(01):5-30

7 Zimmerman GA, Weyrich AS. Signal-dependent protein synthesis by activated platelets: new pathways to altered phenotype and function. Arterioscler Thromb Vasc Biol 2008;28(03):s17-s24

8 Hottz ED, Lopes JF, Freitas C, et al. Platelets mediate increased endothelium permeability in dengue through NLRP3-inflammasome activation. Blood 2013;122(20):3405-3414

9 Lindemann S, Tolley ND, Dixon DA, et al. Activated platelets mediate inflammatory signaling by regulated interleukin $1 \beta$ synthesis. J Cell Biol 2001;154(03):485-490

10 Vinholt PJ, Hvas AM, Frederiksen H, Bathum L, Jørgensen MK, Nybo M. Platelet count is associated with cardiovascular disease, cancer and mortality: a population-based cohort study. Thromb Res 2016;148:136-142

11 Kunicki TJ, Nugent DJ. The genetics of normal platelet reactivity. Blood 2010;116(15):2627-2634

12 O'Donnell CJ, Larson MG, Feng D, et al; Framingham Heart Study. Genetic and environmental contributions to platelet aggregation: the Framingham heart study. Circulation 2001;103(25):3051-3056

13 Crown J, Jakubowski A, Kemeny N, et al. A phase I trial of recombinant human interleukin- 1 beta alone and in combination with myelosuppressive doses of 5-fluorouracil in patients with gastrointestinal cancer. Blood 1991;78(06):1420-1427

14 Kimura H, Ishibashi T, Shikama Y, et al. Interleukin-1 beta (IL-1 beta) induces thrombocytosis in mice: possible implication of IL6. Blood 1990;76(12):2493-2500

15 Netea MG, Joosten LA, Li Y, et al. Understanding human immune function using the resources from the Human Functional Genomics Project. Nat Med 2016;22(08):831-833

16 Ter Horst R, Jaeger M, Smeekens SP, et al. Host and environmental factors influencing individual human cytokine responses. Cell 2016;167(04):1111-1124.e13

17 Aguirre-Gamboa R, Joosten I, Urbano PCM, et al. Differential effects of environmental and genetic factors on $\mathrm{T}$ and $\mathrm{B}$ cell immune traits. Cell Reports 2016;17(09):2474-2487

18 Oosting M, Kerstholt M, Ter Horst R, et al. Functional and genomic architecture of Borrelia burgdorferi-induced cytokine responses in humans. Cell Host Microbe 2016;20(06):822-833

19 Tunjungputri RN, Van Der Ven AJ, Schonsberg A, et al. Reduced platelet hyperreactivity and platelet-monocyte aggregation in HIV-infected individuals receiving a raltegravir-based regimen. AIDS 2014;28(14):2091-2096 
20 Snoep JD, Roest M, Barendrecht AD, De Groot PG, Rosendaal FR Van Der Bom JG. High platelet reactivity is associated with myocardial infarction in premenopausal women: a populationbased case-control study. J Thromb Haemost 2010;8(05): 906-913

21 Tunjungputri RN, van der Ven AJ, Riksen N, et al. Differential effects of platelets and platelet inhibition by ticagrelor on TLR2and TLR4-mediated inflammatory responses. Thromb Haemost 2015;113(05):1035-1045

22 Consortium GOTN; Genome of the Netherlands Consortium. Whole-genome sequence variation, population structure and demographic history of the Dutch population. Nat Genet 2014; 46(08):818-825

23 Deelen P, Bonder MJ, van der Velde KJ, et al. Genotype harmonizer: automatic strand alignment and format conversion for genotype data integration. BMC Res Notes 2014;7:901

24 Delaneau O, Zagury J-F, Marchini J. Improved whole-chromosome phasing for disease and population genetic studies. Nat Methods 2013;10(01):5-6

25 Howie B, Marchini J, Stephens M. Genotype imputation with thousands of genomes. G3 (Bethesda) 2011;1(06):457-470

26 Deelen P, Menelaou A, van Leeuwen EM, et al; Genome of Netherlands Consortium. Improved imputation quality of low-frequency and rare variants in European samples using the 'Genome of The Netherlands'. Eur J Hum Genet 2014;22(11):1321-1326

27 Biino G, Santimone I, Minelli C, et al. Age- and sex-related variations in platelet count in Italy: a proposal of reference ranges based on 40987 subjects' data. PLoS One 2013;8(01):e54289

28 Yeung D, Ciotti S, Purushothama S, et al. Evaluation of highly sensitive immunoassay technologies for quantitative measurements of sub-pg/mL levels of cytokines in human serum. J Immunol Methods 2016;437:53-63

29 Allam O, Samarani S, Jenabian M-A, et al. Differential synthesis and release of IL-18 and IL-18 binding protein from human platelets and their implications for HIV infection. Cytokine 2017;90:144-154

30 Banks RE, Forbes MA, Kinsey SE, et al. Release of the angiogenic cytokine vascular endothelial growth factor (VEGF) from platelets: significance for VEGF measurements and cancer biology. Br J Cancer 1998;77(06):956-964

31 Pott GB, Chan ED, Dinarello CA, Shapiro L. $\alpha$-1-antitrypsin is an endogenous inhibitor of proinflammatory cytokine production in whole blood. J Leukoc Biol 2009;85(05):886-895

32 Jonigk D, Al-Omari M, Maegel L, et al. Anti-inflammatory and immunomodulatory properties of $\alpha 1$-antitrypsin without inhibition of elastase. Proc Natl Acad Sci U S A 2013;110(37): 15007-15012

33 Joosten LA, Crişan TO, Azam T, et al. Alpha-1-anti-trypsin-Fc fusion protein ameliorates gouty arthritis by reducing release and extracellular processing of IL-1 $\beta$ and by the induction of endogenous IL-1Ra. Ann Rheum Dis 2016;75(06):1219-1227

34 Abecassis A, Schuster R, Shahaf G, et al. $\alpha 1$-antitrypsin increases interleukin-1 receptor antagonist production during pancreatic islet graft transplantation. Cell Mol Immunol 2014;11(04): 377-386

35 Jones CI, Bray S, Garner SF, et al; Bloodomics Consortium. A functional genomics approach reveals novel quantitative trait loci associated with platelet signaling pathways. Blood 2009; 114(07):1405-1416

36 Johnson AD. The genetics of common variation affecting platelet development, function and pharmaceutical targeting. J Thromb Haemost 2011;9(Suppl 1):246-257

37 Soranzo N, Spector TD, Mangino M, et al. A genome-wide metaanalysis identifies 22 loci associated with eight hematological parameters in the HaemGen consortium. Nat Genet 2009;41(11): $1182-1190$

38 Lo KS, Wilson JG, Lange LA, et al. Genetic association analysis highlights new loci that modulate hematological trait variation in
Caucasians and African Americans. Hum Genet 2011;129(03): 307-317

39 Schick UM, Jain D, Hodonsky CJ, et al. Genome-wide association study of platelet count identifies ancestry-specific loci in Hispanic/Latino Americans. Am J Hum Genet 2016;98(02):229-242

40 Eicher JD, Chami N, Kacprowski T, et al; Global Lipids Genetics Consortium; CARDIoGRAM Exome Consortium; Myocardial Infarction Genetics Consortium. Platelet-Related Variants Identified by Exomechip Meta-analysis in 157,293 Individuals. Am J Hum Genet 2016;99(01):40-55

41 Johnson AD, Yanek LR, Chen M-H, et al. Genome-wide metaanalyses identifies seven loci associated with platelet aggregation in response to agonists. Nat Genet 2010;42(07):608-613

42 Geisler T, Schaeffeler E, Gawaz M, Schwab M. Genetic variation of platelet function and pharmacology: an update of current knowledge. Thromb Haemost 2013;110(05):876-887

43 Qayyum R, Becker LC, Becker DM, et al. Genome-wide association study of platelet aggregation in African Americans. BMC Genet 2015; $16: 58$

44 Faraday N, Yanek LR, Yang XP, et al. Identification of a specific intronic PEAR1 gene variant associated with greater platelet aggregability and protein expression. Blood 2011;118(12):3367-3375

45 Meisinger C, Prokisch H, Gieger C, et al. A genome-wide association study identifies three loci associated with mean platelet volume. Am J Hum Genet 2009;84(01):66-71

46 van de Veerdonk FL, Netea MG. New insights in the immunobiology of IL-1 family members. Front Immunol 2013;4:167

47 Li Y, Oosting M, Smeekens SP, et al. A functional genomics approach to understand variation in vytokine production in humans. Cell 2016;167(04):1099-1110

48 Welter D, MacArthur J, Morales J, et al. The NHGRI GWAS Catalog, a curated resource of SNP-trait associations. Nucleic Acids Res 2014;42(Database issue):D1001-D1006

49 Rondina MT, Weyrich AS, Zimmerman GA. Platelets as cellular effectors of inflammation in vascular diseases. Circ Res 2013;112 (11):1506-1519

50 Beaulieu LM, Lin E, Mick E, et al. Interleukin 1 receptor 1 and interleukin $1 \beta$ regulate megakaryocyte maturation, platelet activation, and transcript profile during inflammation in mice and humans. Arterioscler Thromb Vasc Biol 2014;34(03):552-564

51 Denis MM, Tolley ND, Bunting M, et al. Escaping the nuclear confines: signal-dependent pre-mRNA splicing in anucleate platelets. Cell 2005;122(03):379-391

52 Sedlmayr P, Blaschitz A, Wilders-Truschnig M, Tiran A, Dohr G. Platelets contain interleukin-1 alpha and beta which are detectable on the cell surface after activation. Scand J Immunol 1995;42 (02):209-214

53 Brown GT, Narayanan P, Li W, Silverstein RL, McIntyre TM. Lipopolysaccharide stimulates platelets through an IL-1 $\beta$ autocrine loop. J Immunol 2013;191(10):5196-5203

54 Shashkin PN, Brown GT, Ghosh A, Marathe GK, McIntyre TM. Lipopolysaccharide is a direct agonist for platelet RNA splicing. J Immunol 2008;181(05):3495-3502

55 Furman D, Chang J, Lartigue L, et al. Expression of specific inflammasome gene modules stratifies older individuals into two extreme clinical and immunological states. Nat Med 2017; 23(02):174-184

56 Ghezzi P, Dinarello CA. IL-1 induces IL-1. III. Specific inhibition of IL1 production by IFN-gamma. J Immunol 1988;140(12):4238-4244

57 Dinkla S, van Cranenbroek B, van der Heijden WA, et al. Platelet microparticles inhibit IL-17 production by regulatory $\mathrm{T}$ cells through P-selectin. Blood 2016;127(16):1976-1986

58 Ando Y, Oku T, Tsuji T. Platelets attenuate production of cytokines and nitric oxide by macrophages in response to bacterial endotoxin. Platelets 2016;27(04):344-350

59 Weyrich AS, McIntyre TM, McEver RP, Prescott SM, Zimmerman GA. Monocyte tethering by P-selectin regulates monocyte chemotactic protein-1 and tumor necrosis factor-alpha secretion. 
Signal integration and NF-kappa B translocation. J Clin Invest 1995;95(05):2297-2303

60 Huang Y, Joshi S, Xiang B, et al. Arf6 controls platelet spreading and clot retraction via integrin $\alpha$ Ilb $\beta 3$ trafficking. Blood 2016;127 (11):1459-1467

61 Kim S, Garcia A, Jackson SP, Kunapuli SP. Insulin-like growth factor-1 regulates platelet activation through PI3-Kalpha isoform. Blood 2007;110(13):4206-4213

62 Wieduwilt MJ, Moasser MM. The epidermal growth factor receptor family: biology driving targeted therapeutics. Cell Mol Life Sci 2008;65(10):1566-1584

63 Heldin $\mathrm{C}-\mathrm{H}$. Targeting the PDGF signaling pathway in tumor treatment. Cell Commun Signal 2013;11:97

64 Petzold T, Ruppert R, Pandey D, et al. $\beta 1$ integrin-mediated signals are required for platelet granule secretion and hemostasis in mouse. Blood 2013;122(15):2723-2731
65 Gasparyan AY, Stavropoulos-Kalinoglou A, Mikhailidis DP, Douglas KM, Kitas GD. Platelet function in rheumatoid arthritis: arthritic and cardiovascular implications. Rheumatol Int 2011; 31(02):153-164

66 Boilard E, Nigrovic PA, Larabee K, et al. Platelets amplify inflammation in arthritis via collagen-dependent microparticle production. Science 2010;327(5965):580-583

67 Hioki H, Aoki N, Kawano K, et al. Acute effects of cigarette smoking on platelet-dependent thrombin generation. Eur Heart J 2001;22 (01):56-61

68 Hung J, Lam JY, Lacoste L, Letchacovski G. Cigarette smoking acutely increases platelet thrombus formation in patients with coronary artery disease taking aspirin. Circulation 1995;92(09):2432-2436

69 Samocha-Bonet D, Justo D, Rogowski O, et al. Platelet counts and platelet activation markers in obese subjects. Mediators Inflamm 2008;2008:834153 